Annals of Pure and Applied Mathematics

Vol. 14, No. 2, 2017, 213-223

ISSN: 2279-087X (P), 2279-0888(online)

Published on 28 August 2017

Annals of

www.researchmathsci.org

DOI: http://dx.doi.org/10.22457/apam.v14n2a2

Pure and Applied

Mathematics

\title{
Separation Axioms and Graphs of Functions in Nano Topological Spaces via Nano $\beta$-open Sets
}

\author{
M. K. Ghosh \\ Department of Mathematics, Kalyani Mahavidyalaya, Kalyani-741235, Nadia \\ West Bengal, India. Email: manabghosh@gmail.com \\ Received 28 July 2017; accepted 19 August 2017
}

Abstract. In this paper, we have introduced some new separation axioms in nano topological spaces in terms of nano $\beta$-open sets along with their basic properties. Two new types of graphs, viz. nano $\beta$-closed graphs and strongly nano $\beta$-closed graphs of functions between two nano topological spaces are initiated in terms of nano $\beta$-open sets. We have established some characterizations of functions having these type of graphs. Moreover, some applications of these graphs on the separation axioms defined here are also achieved.

Keywords: nano $\beta$-closure, $n \beta$ - $T_{i}(i=1,2)$ spaces; $n \beta$-Urysohn, nano $\beta$-closed graph, strongly nano $\beta$-closed graph

AMS Mathematics Subject Classification (2010): 54A05, 54C10, 54D10

\section{Introduction}

The concept of nano topology and nano open sets were introduced by Thivagar [4] in terms of approximations and boundary region of a universal set using an equivalence relation on it. Some recent works on nano topological spaces can be found in $[5,7,10,11]$. Beside these, Nasef et al. [8] have investigated some of the properties of nano near open sets and nano continuity and have shown some application examples in nano topology in real life situation. Recently, Azzam [3] have introduced the concept of grill in nano topological spaces and discussed about some usefulness of nano topology. On the other hand, Monsef et al. [1] introduced the notion of $\beta$-open sets (=semi-preopen sets [2]) and since its introduction such sets along with some of their relevant concepts have been investigated by many researcher.

In the present paper, we have introduced nano $\beta$-closures and which have been used in investigating certain concepts developed in the subsequent sections. In section 4 , some new separation axioms have been introduced in a nano topological space using nano $\beta$-open sets along with various characterizations and properties. Furthermore, in the last two sections, two new types of functions, namely nano $\beta$-closed graph and strongly nano $\beta$-closed graph have been introduced between two nano topological spaces. Some characterizations and basic properties along with possible applications of such functions 


\section{M.K.Ghosh}

are also investigated.

\section{Preliminaries}

Let $\Omega$ be a nonempty finite set called the universe and $R$ be an equivalence relation on $\Omega$. Then the pair $(\Omega, R)$ is called an approximation space. The equivalence of $x \in \Omega$ is denoted by $R(x)$. Let $X \in P(\Omega)$. Then we define the sets

$$
L_{R}(X)=\bigcup_{x \in \Omega}\{R(x): R(x) \subset X\}, H_{R}(X)=\bigcup_{x \in \Omega}\{R(x): R(x) \cap X \neq \varnothing\}
$$

and $B_{R}(X)=L_{R}(X)-H_{R}(X)$. Here the sets $L_{R}(X), H_{R}(X)$ and $B_{R}(X)$ are called lower approximation of $\left(\Omega, \tau_{R}(X)\right)$, upper approximation of $\left(\Omega, \tau_{R}(X)\right)$ and boundary region of $X$ with respect to $R$ respectively. Then $\tau_{R}=\left\{\Omega, \varnothing, H_{R}(X)\right.$, $\left.B_{R}(X), L_{R}(X)\right\}$ is a topology on $\Omega$ with base $\tau_{R}(X)=\left\{\Omega, L_{R}(X), B_{R}(X)\right\}$ [4]. This topology is called a nano topology with respect to the subset $\left(\Omega, \tau_{R}(X)\right)$ of the universe $\Omega$ and the pair $\left(\Omega, \tau_{R}(X)\right)$ is called a nano topological space with respect to the subset $X$ of the universe $\Omega$. The members of $\tau_{R}(X)$ are called nano open sets [4] and their complements are called nano closed sets [4]. Let $A$ be a subset of a nano topological space $\left(\Omega, \tau_{R}(X)\right)$. Then the largest nano open set contained in $A$ is called the nano interior of $A$ [4] and is denoted by $\operatorname{nint}(A)$ and the smallest nano closed set containing $A$ is called the nano closure of $A[4]$ and is denoted by $n c l(A)$.

A subset $A$ of a nano topological space $\left(\Omega, \tau_{R}(X)\right)$ is called nano $\beta$-open [9] if $A \subset \operatorname{ncl}(\operatorname{nint}(\operatorname{ncl}(A)))$. The family of all nano $\beta$-open subsets of a nano topological space $\left(\Omega, \tau_{R}(X)\right)$ is denoted by $N \beta O(\Omega, R, X)=N \beta O(\Omega, X)$. The family of all nano $\beta$-open subsets of a nano topological space $\left(\Omega, \tau_{R}(X)\right)$ containing $x \in \Omega$ is denoted by $N \beta O(\Omega, R, X ; x)=N \beta O(\Omega, X ; x)$. The complement of a nano $\beta$-open set is called a nano $\beta$-closed set. The family of all nano $\beta$-closed subsets of a nano topological space $\left(\Omega, \tau_{R}(X)\right)$ is denoted by $N \beta C(\Omega, R, X)=N \beta C(\Omega, X)$.

\section{Nano $\beta$-closure operators}

Some of the concepts and results developed here will be used in the subsequent sections.

Definition 3.1. A nano topological space $\left(\Omega, \tau_{R}(X)\right)$ is said to satisfy a property $n P$ if $n c l(A \cap B)=n c l(A) \cap n c l(B)$ for every pair of subsets $A$ and $B$ of a nano topological space $\left(\Omega, \tau_{R}(X)\right)$.

Theorem 3.2. (a) Arbitrary union of nano $\beta$-open sets of a nano topological space $\left(\Omega, \tau_{R}(X)\right)$ is a nano $\beta$-open set.

(b) If a nano topological space $\left(\Omega, \tau_{R}(X)\right)$ satisfies the property $n P$, then the intersection of any two nano $\beta$-open sets is nano $\beta$-open and so $n \beta O(\Omega, X)$ is a 
Separation Axioms and Graphs of Functions in Nano Topological Spaces via Nano

$\beta$-open Sets

topology on $\Omega$ finer than nano topology $\tau_{R}(X)$.

Proof: (a): Obvious.

(b) Let $A$ and $B$ are any two nano $\beta$-open sets. Then $A \subset \operatorname{ncl}(\operatorname{nint}(\operatorname{ncl}(A)))$ and $B \subset n c l(\operatorname{nint}(n c l(B)))$.

Now $A \cap B \subset \operatorname{ncl}(\operatorname{nint}(n c l(B)))=n c l(\operatorname{nint}(n c l(A)) \cap \operatorname{nint}(\operatorname{ncl}(B)))$

$=n c l(\operatorname{nint}(n c l(A) \cap n c l(B)))=n c l(\operatorname{nint}(n c l(A \cap B)))$ and so $A \cap B$ is nano $\beta$-open.

Definition 3.3. Let $A$ be a subset of a nano topological space $\left(\Omega, \tau_{R}(X)\right)$. Then $n \beta$ -interior (resp. $n \beta$-closure) of $A$ is denoted by $n \beta \operatorname{int}(A)$ (respectively $n \beta c l(A)$ ) and is defined as the set $n \beta \operatorname{int}(A)=\cup\{G \subset A: G \in n \beta O(\Omega, X)\}$ (respetively $n \beta c l(A)=\cap\{F \supset A: \Omega-F \in n \beta O(\Omega, X)\})$.

Theorem 3.4. For any subsets $A$ and $B$ of a nano topological space $\left(\Omega, \tau_{R}(X)\right)$, the following statements hold:

(i) $x \in n \beta c l(A)$ if and only if $A \cap U \neq \varnothing$ for each $U \in N \beta O(\Omega, X ; x)$;

(ii) $A$ is nano $\beta$-open if and only if $A=n \beta \operatorname{int}(A)$;

(iii) $A$ is nano $\beta$-closed if and only if $A=n \beta c l(A)$;

(iv) If $A \subset B$ then $n \beta \operatorname{int}(A) \subset n \beta \operatorname{int}(B)$ and $n \beta c l(A) \subset n \beta c l(B)$;

(v) $n \beta c l(\Omega-A)=\Omega-n \beta \operatorname{int}(A)$;

(vi) $\operatorname{nint}(A) \subset n \beta \operatorname{int}(A)$;

(vii) $n c l(A) \supset n \beta c l(A)$.

Proof: Straightforward.

\section{Separation axioms in terms of nano $\beta$-open sets}

Definition 4.1. A space $\left(\Omega, \tau_{R}(X)\right)$ is called

(i) $n \beta-T_{1}$ if for each pair of distinct points $x, y \in \Omega$, there exist an

$U_{x} \in N \beta O(\Omega, X ; x)$ and an $U_{y} \in N \beta O(\Omega, X ; y)$ such that $x \notin U_{y}$ and $y \notin U_{x}$.

(ii) $n \beta-T_{2}$ if for each pair of distinct points $x, y \in \Omega$, there exist an $U_{x} \in N \beta O(\Omega, X ; x)$ and an $U_{y} \in N \beta O(\Omega, X ; y)$ such that $U_{x} \cap U_{y}=\varnothing$.

(iii) $n \beta$-Urysohn if for each pair of distinct points $x, y \in \Omega$, there exist an $U_{x} \in N \beta O(\Omega, X ; x)$ and an $U_{y} \in N \beta O(\Omega, X ; y)$ such that

$n \beta \operatorname{cl}\left(U_{x}\right) \cap n \beta c l\left(U_{y}\right)=\varnothing$.

(iv) $n \beta$-regular if for each point $x \in \Omega$ and each nano $\beta$-closed set $F$ such that $x \notin F$, there exist a $V \in N \beta O(\Omega, X ; x)$ and a $W \in N \beta O(\Omega, X)$ such that $F \subset W$ and $V \cap W=\varnothing$. 


\section{M.K.Ghosh}

Remark 4.2. A $n \beta$-Urysohn nano topological space is a $n \beta-T_{2}$ nano topological space and a $n \beta-T_{2}$ nano topological space is a $n \beta-T_{1}$ nano topological space.

The following characterizations of $n \beta-T_{1}, n \beta-T_{2}$ and $n \beta$-regular spaces are straightforward.

Theorem 4.3. A nano topological space $\left(\Omega, \tau_{R}(X)\right)$ is $n \beta-T_{1}$ if and only if singletons of $\left(\Omega, \tau_{R}(X)\right)$ are nano $\beta$-closed.

Theorem 4.4. For a nano topological space $\left(\Omega, \tau_{R}(X)\right)$, the following statements are equivalent:

(a) $\left(\Omega, \tau_{R}(X)\right)$ is $n \beta-T_{2}$;

(b) For each $x \in \Omega$ and $y(\neq x) \in \Omega$, there exist an $U_{x} \in N \beta O(\Omega, X ; x)$ such that $y \notin n \beta c l\left(U_{x}\right)$.

(c) For each $x \in \Omega, \cap\{n \beta c l(U): U \in n \beta O(\Omega, X ; x)\}=\{x\}$.

Theorem 4.5. A nano topological space $\left(\Omega, \tau_{R}(X)\right)$ is $n \beta$-regular if and only if for each $x \in \Omega$ and each $U \in N \beta O(\Omega, X ; x)$, there exists $a V \in N \beta O(\Omega, X ; x)$ such that $n \beta-\operatorname{cl}(V) \subset U$.

Theorem 4.6. Every $n \beta$-regular $n \beta-T_{2}$ nano topological space is $n \beta$-Urysohn.

Proof: Let the nano topological space $\left(\Omega, \tau_{R}(X)\right)$ is $n \beta$-regular and $n \beta-T_{2}$. Consider any two distinct points $x, y \in \Omega$. Since $\left(\Omega, \tau_{R}(X)\right)$ is $n \beta-T_{2}$, there exist an $U_{x} \in n \beta O(\Omega, X ; x)$ and an $U_{y} \in n \beta O(\Omega, X ; y)$ such that $U_{x} \cap U_{y}=\varnothing$ and so $n \beta c l\left(U_{x}\right) \cap U_{y}=\varnothing$. Then $U=\Omega-n \beta c l\left(U_{x}\right) \in n \beta O(\Omega, X ; y)$. Since $\left(\Omega, \tau_{R}(X)\right)$ is $n \beta$-regular, by Theorem 4.5 , we can find a $V_{y} \in n \beta O(\Omega, X ; y)$ such that $n \beta c l\left(V_{y}\right) \subset U$. Thus $n \beta c l\left(V_{y}\right) \cap n \beta c l\left(U_{x}\right)=\varnothing$. So $\left(\Omega, \tau_{R}(X)\right)$ is $n \beta$-Urysohn.

Definition 4.7. Let $\left(\Omega, \tau_{R}(X)\right)$ and $\left(\Lambda, \tau_{R^{\star}}(Y)\right)$ be two nano topological spaces. Then a function $\psi: \Omega \rightarrow \Lambda$ is called nano $\beta$-open if $\psi(U) \in N \beta O\left(\Lambda, R^{\star}, Y\right)$ for every $U \in N \beta O(\Omega, R, X)$.

Remark 4.8. Let $\left(\Omega, \tau_{R}(X)\right)$ and $\left(\Lambda, \tau_{R^{\star}}(Y)\right)$ be two nano topological spaces. Then a surjection $\psi: \Omega \rightarrow \Lambda$ is nano $\beta$-open if and only if $\psi(U) \in N \beta C\left(\Lambda, R^{\star}, Y\right)$ for every $U \in N \beta C(\Omega, R, X)$. 
Separation Axioms and Graphs of Functions in Nano Topological Spaces via Nano

$\beta$-open Sets

Definition 4.9. Let $\left(\Omega, \tau_{R}(X)\right)$ and $\left(\Lambda, \tau_{R^{\star}}(Y)\right)$ be two nano topological spaces. Then a function $\psi: \Omega \rightarrow \Lambda$ is called quasi nano $\beta$-irresolute if for each $x \in \Omega$ and for each $V \in N \beta O\left(\Lambda, R^{\star}, Y ; \psi(x)\right)$, there exists an $U \in N \beta O(\Omega, R, X ; x)$ such that $\psi(U) \subset n \beta c l(V)$.

Theorem 4.10. Let $\left(\Omega, \tau_{R}(X)\right)$ and $\left(\Lambda, \tau_{R^{\star}}(Y)\right)$ be two nano topological spaces. Let $\psi: \Omega \rightarrow \Lambda$ be a quasi nano $\beta$-irresolute and injective mapping, where $\Lambda$ be $n \beta$ -Urysohn. Then $\left(\Omega, \tau_{R}(X)\right)$ is $n \beta-T_{2}$.

Proof: Let $p_{1}$ and $p_{2}$ be any two distinct points of $\left(\Omega, \tau_{R}(X)\right)$. Since $\psi$ is injective, $\psi\left(p_{1}\right) \neq \psi\left(p_{2}\right)$. Again since $\Lambda$ is $n \beta$-Urysohn, there exist a $V_{p_{1}} \in N \beta O\left(\Lambda, R^{\star}\right.$, $\left.Y ; \psi\left(p_{1}\right)\right)$ and a $V_{p_{2}} \in N \beta O\left(\Lambda, R^{\star}, Y ; \psi\left(p_{2}\right)\right)$ such that $n \beta c l\left(V_{p_{1}}\right) \cap n \beta c l\left(V_{p_{2}}\right)=\varnothing$. Also since $\psi$ is quasi nano $\beta$-irresolute, there exist a $S_{p_{1}} \in N \beta O\left(\Omega, R, X ; p_{1}\right)$ and a $S_{p_{2}} \in N \beta O\left(\Omega, R, X ; p_{2}\right)$ such that $\psi\left(S_{p_{1}}\right) \subset n \beta c l\left(V_{p_{1}}\right)$ and $\psi\left(S_{p_{2}}\right) \subset n \beta c l\left(V_{p_{2}}\right)$ and hence $\psi\left(S_{p_{1}}\right) \cap \psi\left(S_{p_{2}}\right) \subset n \beta c l\left(V_{p_{1}}\right) \cap n \beta c l\left(V_{p_{2}}\right)=\varnothing$. Thus $\psi\left(S_{p_{1}}\right) \cap \psi\left(S_{p_{2}}\right)=\varnothing$ and so $S_{p_{1}} \cap S_{p_{2}}=\varnothing$. Therefore $\left(\Lambda, \tau_{R^{\star}}(Y)\right)$ is $n \beta-T_{2}$.

Theorem 4.11. Let $\left(\Omega, \tau_{R}(X)\right)$ and $\left(\Lambda, \tau_{R^{\star}}(Y)\right)$ be two nano topological spaces. Let the function $\psi: \Omega \rightarrow \Lambda$ be nano $\beta$-open and bijective, where $\left(\Omega, \tau_{R}(X)\right)$ be $n \beta$ -Urysohn. Then $\Lambda$ is $n \beta$-Urysohn.

Proof: Let $q_{1}$ and $q_{2}$ be any two distinct points of $\Lambda$. Since $\psi$ is bijective, there exist $p_{1}, p_{2} \in \Omega$ with $p_{1} \neq p_{2}, \psi\left(p_{1}\right)=q_{1}$ and $\psi\left(p_{2}\right)=q_{2}$. Since $\left(\Omega, \tau_{R}(X)\right)$ is $n \beta$ -Urysohn, there exist a $S_{p_{1}} \in N \beta O\left(\Omega, R, X ; p_{1}\right)$ and a $S_{p_{2}} \in N \beta O\left(\Omega, R, X ; p_{2}\right)$ such that $n \beta c l\left(S_{p_{1}}\right) \cap n \beta c l\left(S_{p_{2}}\right)=\varnothing$. But the nano $\beta$-open-ness of $\psi$ ensures that $\psi\left(\Omega-n \beta c l\left(S_{p_{1}}\right)\right)=\Lambda-\psi\left(n \beta c l\left(S_{p_{1}}\right)\right)$ and $\psi\left(\Omega-n \beta c l\left(S_{p_{2}}\right)\right)=\Lambda-\psi\left(n \beta c l\left(S_{p_{2}}\right)\right)$ are nano $\beta$-open sets and so $\psi\left(n \beta c l\left(S_{p_{1}}\right)\right)$ and $\psi\left(n \beta c l\left(S_{p_{2}}\right)\right)$ are $n \beta$-closed in $\left(\Lambda, \tau_{R^{\star}}(Y)\right)$.

Now $n \beta c l\left(\psi\left(S_{p_{1}}\right)\right) \cap n \beta c l\left(\psi\left(S_{p_{2}}\right)\right) \subset n \beta c l\left(\psi\left(n \beta c l\left(S_{p_{1}}\right)\right)\right) \cap n \beta c l\left(\psi\left(n \beta c l\left(S_{p_{2}}\right)\right)\right)$ $=\psi\left(n \beta c l\left(S_{p_{1}}\right)\right) \cap \psi\left(n \beta c l\left(S_{p_{2}}\right)\right)=\psi\left(n \beta c l\left(S_{p_{1}}\right) \cap n \beta c l\left(S_{p_{2}}\right)\right)=\varnothing$. Also, since $\psi$ is nano $\beta$-open, $\psi\left(S_{p_{1}}\right) \in n \beta O\left(\Lambda, R^{\star}, Y ; q_{1}\right)$ and $\psi\left(S_{p_{2}}\right) \in N \beta O\left(\Lambda, R^{\star}, Y ; q_{2}\right)$. Thus 


\section{M.K.Ghosh}

$\left(\Lambda, \tau_{R^{\star}}(Y)\right)$ is $N \beta$-Urysohn.

\section{Nano $\beta$-closed and strongly nano $\beta$-closed graphs}

Definition 5.1. Let $\left(\Omega, \tau_{R}(X)\right)$ and $\left(\Lambda, \tau_{R^{\star}}(Y)\right)$ be two nano topological spaces and $\psi: \Omega \rightarrow \Lambda$ be a function. Then its graph $G(\psi)$ is called nano $\beta$-closed if for each $(x, y) \in \Omega \times \Lambda-G(\psi)$, there exist an $U \in N \beta O(\Omega, X ; x)$ and a $V \in N \beta O\left(\Lambda, R^{\star}, Y ; y\right)$ such that $(U \times V) \cap G(\psi)=\varnothing$.

Lemma 5.2. Let $\left(\Omega, \tau_{R}(X)\right)$ and $\left(\Lambda, \tau_{R^{\star}}(Y)\right)$ be two nano topological spaces and $\psi: \Omega \rightarrow \Lambda$ be a function. Then following conditions are equivalent.

(i) $\psi$ has nano $\beta$-closed graph,

(ii) for each $(x, y) \in \Omega \times \Lambda-G(\psi)$, there exist an $U \in N \beta O(\Omega, R, X ; x)$ and a $V \in N \beta O\left(\Lambda, R^{\star}, Y ; y\right)$ such that $\psi(U) \cap V=\varnothing$.

Proof: The proof is straightforward and thus omitted.

Definition 5.3. Let $\left(\Omega, \tau_{R}(X)\right)$ and $\left(\Lambda, \tau_{R^{\star}}(Y)\right)$ be two nano topological spaces. A function $\psi: \Omega \rightarrow \Lambda$ is called nano $\beta$-irresolute if for each $x \in \Omega$ and for each $V \in N \beta O\left(\Lambda, R^{\star}, Y ; \psi(x)\right)$, there exists an $U \in N \beta O(\Omega, R, X ; x)$ such that $\psi(U) \subset V$.

Theorem 5.4. Let $\left(\Omega, \tau_{R}(X)\right),\left(\Lambda, \tau_{R^{\star}}(Y)\right)$ be two nano topological spaces and $\psi: \Omega \rightarrow \Lambda$ be nano $\beta$-irresolute, where $\Lambda$ is $n \beta-T_{2}$. Then the graph $G(\psi)$ is nano $\beta$-closed.

Proof: Let $(x, y) \in \Omega \times \Lambda-G(\psi)$. Then $y \neq \psi(x)$. Since $\Lambda$ is $n \beta-T_{2}$, there exist an $U_{1} \in N \beta O\left(\Lambda, R^{\star}, Y ; \psi(x)\right)$ and a $V \in N \beta O\left(\Lambda, R^{\star}, Y ; y\right)$ such that $U_{1} \cap V=\varnothing$. Also, since $\psi$ is nano $\beta$-irresolute, $U=\psi^{-1}\left(U_{1}\right) \in N \beta O(\Omega, R, X ; x)$ and so $\psi(U) \cap V=\varnothing$. Therefore the graph $G(\psi)$ is nano $\beta$-closed.

Theorem 5.5. Let $\left(\Omega, \tau_{R}(X)\right),\left(\Lambda, \tau_{R^{\star}}(Y)\right)$ be two nano topological spaces and $\psi: \Omega \rightarrow \Lambda$ be a surjective mapping having nano $\beta$-closed graph. Then $\left(\Lambda, \tau_{R^{\star}}(Y)\right)$ is $n \beta-T_{1}$.

Proof: Let $q_{1}, q_{2} \in \Lambda$ with $q_{1} \neq q_{2}$. Since $\psi$ is surjective, there exists $p_{1} \in \Omega$ such that $\psi\left(p_{1}\right)=q_{1}$ and $\psi\left(p_{1}\right) \neq q_{2}$. Then $\left(p_{1}, q_{2}\right) \in \Omega \times \Lambda-G(\psi)$ and so by the Lemma 5.2, we can find $U_{p_{1}} \in N \beta O\left(\Omega, R, X ; p_{1}\right)$ and a $V_{q_{2}} \in N \beta O\left(\Lambda, R^{\star}, Y ; q_{2}\right)$ such that $\psi\left(U_{p_{1}}\right) \cap V_{q_{2}}=\varnothing$. Thus $q_{1} \in \psi\left(U_{p_{1}}\right)$ and so $q_{1} \notin V_{q_{2}}$. Similarly, we can ensure the 
Separation Axioms and Graphs of Functions in Nano Topological Spaces via Nano

$\beta$-open Sets

existence of an $p_{2} \in \Omega$ such that $\psi\left(p_{2}\right)=q_{2} \quad$ and $\psi\left(p_{2}\right) \neq q_{1}$ and an $U_{p_{2}} \in N \beta O\left(\Omega, R, X ; p_{2}\right)$ and a $V_{q_{1}} \in N \beta O\left(\Lambda, R^{\star}, Y ; q_{1}\right)$ such that $\psi\left(U_{p_{2}}\right) \cap V_{q_{1}}=\varnothing$. Then $q_{2} \in \psi\left(U_{p_{2}}\right)$ and $q_{2} \notin V_{q_{1}}$. So $\left(\Lambda, \tau_{R^{\star}}(Y)\right)$ is $n \beta-T_{1}$.

Theorem 5.6. Let $\left(\Omega, \tau_{R}(X)\right)$, $\left(\Lambda, \tau_{R^{\star}}(Y)\right)$ be two nano topological spaces and the function $\psi: \Omega \rightarrow \Lambda$ be nano $\beta$-open and surjective. If the graph $G(\psi)$ is $\beta$-closed, then $\left(\Lambda, \tau_{R^{\star}}(Y)\right)$ is $n \beta-T_{2}$.

Proof: Let $\left(\Omega, \tau_{R}(X)\right)$ and $\left(\Lambda, \tau_{R^{\star}}(Y)\right)$ be two nano topological spaces. Let $q_{1}, q_{2} \in \Lambda$ with $q_{1} \neq q_{2}$. Since $\psi$ is surjective, there exist $p_{1} \in \Omega$ such that $\psi\left(p_{1}\right)=q_{1}$ but $\psi\left(p_{1}\right) \neq q_{2}$. Thus $\left(p_{1}, q_{2}\right) \in \Omega \times \Lambda-G(\psi)$ and so by Lemma 5.2, we can find an $U_{p_{1}} \in N \beta O\left(\Omega, R, X ; p_{1}\right)$ and a $V_{q_{2}} \in N \beta O\left(\Lambda, R^{\star}, Y ; q_{2}\right)$ such that $\psi\left(U_{p_{1}}\right) \cap V_{q_{2}}=\varnothing$. Again since $\psi$ is nano $\beta$-open, $\psi\left(U_{p_{1}}\right) \in N \beta O\left(\Lambda, R^{\star}, Y ; q_{1}\right)$. So $\left(\Lambda, \tau_{R^{\star}}(Y)\right)$ is $n \beta-T_{2}$.

Theorem 5.7. Let $\left(\Omega, \tau_{R}(X)\right),\left(\Lambda, \tau_{R^{\star}}(Y)\right)$ be two nano topological spaces and $\psi: \Omega \rightarrow \Lambda$ be injective. If the graph $G(\psi) \beta$-closed, $\left(\Omega, \tau_{R}(X)\right)$ is $n \beta-T_{1}$.

Proof: Let $p_{1}, p_{2} \in \Omega$ and $p_{1} \neq p_{2}$. Since $\psi$ is injective, $\psi\left(p_{1}\right) \neq \psi\left(p_{2}\right)$.

So $\left(p_{1}, \psi\left(p_{2}\right)\right) \in \Omega \times \Lambda-G(\psi)$. Now, by Lemma 5.2, there exist an $U_{p_{1}} \in N \beta O\left(\Omega, R, X ; p_{1}\right)$ and a $V_{p_{2}} \in N \beta O\left(\Lambda, R^{\star}, Y ; \psi\left(p_{2}\right)\right)$ such that $\psi\left(U_{p_{1}}\right) \cap V_{p_{2}}=\varnothing$. Therefore $\psi\left(p_{2}\right) \notin\left(U_{p_{1}}\right)$ and so $p_{2} \notin U_{p_{1}}$. Hence $p_{1} \in U_{p_{1}}$ but $p_{2} \notin U_{p_{1}}$. Again since $\left(p_{2}, \psi\left(p_{1}\right)\right) \in \Omega \times \Lambda-G(\psi)$, we can find $U_{p_{2}} \in N \beta O\left(\Omega, R, X ; p_{2}\right)$ and $p_{1} \notin U_{p_{2}}$. Hence $\Omega$ is $n \beta-T_{1}$.

Theorem 5.8. Let $\left(\Omega, \tau_{R}(X)\right),\left(\Lambda, \tau_{R^{\star}}(Y)\right)$ be two nano topological spaces and $\psi: \Omega \rightarrow \Lambda$ be a nano $\beta$-irresolute injection. If the graph $G(\psi)$ is $\beta$-closed, $\left(\Omega, \tau_{R}(X)\right)$ is $n \beta-T_{2}$.

Proof: Let $p_{1}, p_{2} \in \Omega$ and $p_{1} \neq p_{2}$. Since $\psi$ is injective, $\psi\left(p_{1}\right) \neq \psi\left(p_{2}\right)$. So $\left(p_{1}, \psi\left(p_{2}\right)\right) \in \Omega \times \Lambda-G(\psi)$. Then Lemma 5.2 ensures the existence of an $U_{p_{1}} \in N \beta O\left(\Omega, R, X ; p_{1}\right)$ and a $V_{p_{2}} \in N \beta O\left(\Lambda, R^{\star}, Y ; \psi\left(p_{2}\right)\right)$ such that $\psi\left(U_{p_{1}}\right) \cap V_{p_{2}}=\varnothing$. Since $\psi$ is a nano $\beta$-irresolute, $\psi^{-1}\left(V_{q_{2}}\right) \in N \beta O\left(\Omega, R, X ; p_{2}\right)$. So $\left(\Omega, \tau_{R}(X)\right)$ is $n \beta-T_{2}$. 


\section{M.K.Ghosh}

Definition 5.9. [8] Let $\left(\Omega, \tau_{R}(X)\right)$ and $\left(\Lambda, \tau_{R^{\star}}(Y)\right)$ be two nano topological spaces. A function $\psi: \Omega \rightarrow \Lambda$ is called nano $\beta$-continuous if $\psi^{-1}(V) \in N \beta O(\Omega, R, X)$ for every $V \in N O\left(\Lambda, R^{\star}, Y\right)$.

Theorem 5.10. Let a nano topological space $\left(\Omega, \tau_{R}(X)\right)$ satisfy the property $n P$ and $\left(\Lambda, \tau_{R^{\star}}(Y)\right)$ be an arbitrary nano topological spaces. And $\psi: \Omega \rightarrow \Lambda$ has nano $\beta$ -closed graph. Then $\psi$ is nano $\beta$-continuous.

Proof: Let $V \in N O\left(\Lambda, R^{\star}, Y\right)$ and any $x \in \psi^{-1}(V)$. Then for each $y \in \Lambda-V$, $(x, y) \in \Omega \times \Lambda-G(\psi)$. Since the graph of $\psi$ is nano $\beta$-closed, there exists an $U_{y} \in N \beta O(\Omega, R, X ; x)$ and a $V_{y} \in N \beta O\left(\Lambda, R^{\star}, Y ; y\right)$ such that $\psi\left(U_{y}\right) \cap V_{y}=\varnothing$. Since $\left(\Omega, \tau_{R}(X)\right)$ is finite, we can find $q_{1}, q_{2}, \ldots, y_{k} \in \Lambda-V$ such that $Y=\left(\cup_{i=1}^{k} V_{y_{i}}\right) \cup V$ and so $\Lambda-V \subset \cup_{i=1}^{k} V_{y_{i}}$. Since $\left(\Omega, \tau_{R}(X)\right)$ satisfy the property $n P, S_{x}=\cap_{i=1}^{k} U_{y_{i}} \in N \beta O(\Omega, R, X ; x)$ and $\psi(S) \cap(\Lambda-V)=\varnothing$. So $\psi^{-1}(V)=\cup\left\{S_{x}: x \in \psi^{-1}(V)\right\} \in N \beta O(\Omega, R, X)$. Therefore $\psi$ is nano $\beta$-continuous.

Definition 5.11. Let $\left(\Omega, \tau_{R}(X)\right),\left(\Lambda, \tau_{R^{\star}}(Y)\right)$ be two nano topological spaces and $\psi: \Omega \rightarrow \Lambda$ be a function. Then its graph $G(\psi)$ is called strongly nano $\beta$-closed if for each $(x, y) \in \Omega \times \Lambda-G(\psi)$, there exist an $U \in N \beta O(\Omega, R, X ; x)$ and $a$

$V \in N \beta O\left(\Lambda, R^{\star}, Y ; y\right)$ such that $(U \times n \beta c l(V)) \cap G(\psi)=\varnothing$.

Clearly, every function possessing strongly nano $\beta$-closed graph has nano $\beta$ -closed graph.

Lemma 5.12. Let $\left(\Omega, \tau_{R}(X)\right)$ and $\left(\Lambda, \tau_{R^{\star}}(Y)\right)$ be two nano topological spaces. Then for a function $\psi: \Omega \rightarrow \Lambda$, following conditions are equivalent:

(i) the graph $G(\psi)$ is nano $\beta$-closed;

(ii) for each $(x, y) \in \Omega \times \Lambda-G(\psi)$, there exist an $U \in N \beta O(\Omega, R, X ; x)$ and a $V \in N \beta O\left(\Lambda, R^{\star}, Y ; y\right)$ such that $\psi(U) \cap n \beta c l(V)=\varnothing$.

Proof: The proof is straightforward and so omitted.

Definition 5.13. A filter base $\mathcal{F}$ on a nano topological space $\left(\Omega, \tau_{R}(X)\right)$ is said to nano $\beta$ - $\theta$-converge (respectively nano $\beta$-converge) to a point $x \in \Omega$ if for each $V \in N \beta O(\Omega, R, X ; x)$, there exists an $F \in \mathcal{F}$ such that $F \subset n \beta c l(V)$ (respectively $F \subset V)$.

Theorem 5.14. Let $\left(\Omega, \tau_{R}(X)\right)$ and $\left(\Lambda, \tau_{R^{\star}}(Y)\right)$ be two nano topological spaces. Let $\left(\Lambda, \tau_{R^{\star}}(Y)\right)$ be $n \beta$-regular and $\psi: \Omega \rightarrow \Lambda$ be any function. Then the following 
Separation Axioms and Graphs of Functions in Nano Topological Spaces via Nano

$\beta$-open Sets

statements are equivalent:

(i) $G(\psi)$ is strongly nano $\beta$-closed;

(ii) If a filter base $\mathcal{F}$ on $\left(\Omega, \tau_{R}(X)\right)$, nano $\beta$-converges to $x$ and $\psi(\mathcal{F})$ nano $\beta$ - $\theta$-converges to $y$ in $\left(\Lambda, \tau_{R^{\star}}(Y)\right)$, then $y=\psi(x)$.

Proof: (i) $\Rightarrow$ (ii): Let $\mathcal{F}$ be a filter base on $\left(\Omega, \tau_{R}(X)\right)$ that nano $\beta$-converges to $x$ and $\psi(\mathcal{F})$ nano $\beta$ - $\theta$-converges to $y$. If possible, let $y \neq \psi(x)$.

Then $(x, y) \in \Omega \times \Lambda-G(\psi)$. Clearly, $N \beta O(\Omega, R, X ; x) \subset \mathcal{F}$ and $\left\{n \beta c l(V): V \in N \beta O\left(\Lambda, R^{\star}, Y ; y\right)\right\} \subset \psi(\mathcal{F})$. So, for each $U \in N \beta O(\Omega, R, X ; x)$ and each $V \in N \beta O\left(\Lambda, R^{\star}, Y ; y\right)$, there exist $P_{1} \in \mathcal{F}$ and $P_{2} \in \mathcal{F}$ such that $P_{1} \subset U$ and $\psi\left(P_{2}\right) \in n \beta c l(V)$. Hence there exists an $P_{0} \in \mathcal{F}$ such that $P_{0} \subset P_{1} \cap P_{2}$ and satisfies $P_{0} \subset U$ as well as $\psi\left(P_{0}\right) \subset n \beta c l(V)$. Hence $\varnothing \neq \psi\left(P_{0}\right) \subset \psi(U) \cap n \beta c l(V)$. So by Lemma 5.12, $G(\psi)$ is not strongly nano $\beta$-closed.

(ii) $\Rightarrow$ (i): Let $\left(\Lambda, \tau_{R^{\star}}(Y)\right)$ is $n \beta$-regular and the given condition (ii) holds for $\psi$. If possible, let $G(\psi)$ is not strongly nano $\beta$-closed. Then there exists $(x, y) \in \Omega \times \Lambda-G(\psi) \quad$ such $\quad$ that $\quad(U \times n \beta c l(V)) \cap G(\psi) \neq \varnothing \quad$ for each $U \in N \beta O(\Omega, R, X ; x)$ and each $V \in N \beta O\left(\Lambda, R^{\star}, Y ; y\right)$. Since $\Lambda$ is $n \beta$-regular, the family

$\mathcal{F}=\left\{F_{U V}=\{p \in U:(p, \psi(p)) \in(U \times n \beta c l(V)) \cap G(\psi)\}: U \in N \beta O(\Omega, R, X ; x)\right.$ and $\left.V \in N \beta O\left(\Lambda, R^{\star}, Y ; y\right)\right\}$ is a filter base on $\left(\Omega, \tau_{R}(X)\right)$. But $\mathcal{F}$ nano $\beta$-converges to $x$ in $\left(\Omega, \tau_{R}(X)\right)$ and $\psi(\mathcal{F})$ nano $\beta-\theta$-converges to $y$ and $y=\psi(x)-$ a contradiction.

Theorem 5.15. Let $\left(\Omega, \tau_{R}(X)\right),\left(\Lambda, \tau_{R^{\star}}(Y)\right)$ be two nano topological spaces and $\psi: \Omega \rightarrow \Lambda$ be a function. If the graph $G(\psi)$ is strongly nano $\beta$-closed, then $\psi(x)=\cap\{n \beta c l(\psi(U)): U \in N \beta O(\Omega, R, X ; x)\}$ for each $x \in \Omega$.

Proof: If possible, let there exist an $x \in \Omega$ and an $y(\neq \psi(x)) \in \Lambda$ such that $y \in n \beta c l(\psi(U))$ for each $U \in N \beta O(\Omega, R, X ; x)$. Since $(x, y) \in \Omega \times \Lambda-G(\psi)$, by Lemma 5.12, we can find a $U_{x} \in N \beta O(\Omega, R, X ; x)$ and a $V_{y} \in N \beta O\left(\Lambda, R^{\star}, Y ; y\right)$ such that $\psi\left(U_{x}\right) \cap n \beta c l\left(V_{y}\right)=\varnothing$ and so $\psi\left(U_{x}\right) \cap V_{y} \subset \psi\left(U_{x}\right) \cap n \beta c l\left(V_{y}\right)=\varnothing$. Thus $\psi\left(U_{x}\right) \cap V_{y}=\varnothing$. Then $y \notin n \beta c l\left(\psi\left(U_{x}\right)\right)$, a contradiction.

Theorem 5.16. Let $\left(\Omega, \tau_{R}(X)\right)$ is an arbitrary nano topological space and $\left(\Lambda, \tau_{R^{\star}}(Y)\right)$ is $n \beta-T_{2}$. Let $\psi: \Omega \rightarrow \Lambda$ be nano $\beta$-irresolute. Then the graph $G(\psi)$ is strongly nano $\beta$-closed. 


\section{M.K.Ghosh}

Proof: Let $(x, y) \in \Omega \times \Lambda-G(\psi)$. Then $y \neq \psi(x)$. Since $\left(\Lambda, \tau_{R^{\star}}(Y)\right)$ is $n \beta-T_{2}$, there exist a $U \in N \beta O\left(\Lambda, R^{\star}, Y ; \psi(x)\right)$ and a $V \in N \beta O\left(\Lambda, R^{\star}, Y ; y\right)$ such that $U \cap V=\varnothing$. Again since $\psi$ is nano $\beta$-irresolute, $K=\psi^{-1}(U) \in N \beta O(\Omega, R, X ; x)$ and so $\psi(K) \cap V=\varnothing$, i.e. $V \subset \Lambda-\psi(K)$, i.e. $n \beta c l(V) \subset \Lambda-\psi(K)$, i.e. $\psi(K) \cap n \beta c l(V)=\varnothing$. Hence $G(\psi)$ is strongly nano $\beta$-closed.

Theorem 5.17. Let $\left(\Omega, \tau_{R}(X)\right),\left(\Lambda, \tau_{R^{\star}}(Y)\right)$ be two nano topological spaces and $\psi: \Omega \rightarrow \Lambda$ be a quasi nano $\beta$-irresolute injection. If the graph $G(\psi)$ is strongly nano $\beta$-closed, then $\left(\Omega, \tau_{R}(X)\right)$ is $n \beta-T_{2}$.

Proof: Let any two distinct points $p_{1}, p_{2} \in \Omega$. Since $\psi$ is injective, $\psi\left(p_{1}\right) \neq \psi\left(p_{2}\right)$. Thus $\left(p_{1}, \psi\left(p_{2}\right)\right) \in \Omega \times \Lambda-G(\psi)$ and so by lemma 5.12 , there exist a $U_{p_{1}} \in N \beta O(\Omega, R, X ; x)$ and a $V_{p_{2}} \in N \beta O\left(\Lambda, R^{\star}, Y ; y\right)$ such that $\psi\left(U_{p_{1}}\right) \cap$ $n \beta c l\left(V_{p_{2}}\right)=\varnothing$ and so $\psi^{-1}\left(n \beta c l\left(V_{p_{2}}\right)\right) \subset \Omega-U_{p_{1}}$. Since $\psi$ is a quasi nano $\beta$ -irresolute, there exists $S_{p_{2}} \in(n) \beta \gamma\left(\Omega, R, X ; p_{2}\right)$ such that $\psi\left(S_{p_{2}}\right) \in n \beta c l\left(V_{p_{2}}\right)$. Then $S_{p_{2}} \subset \psi^{-1}\left(n \beta c l\left(V_{p_{2}}\right)\right) \subset \Omega-U_{p_{1}}$ and hence $S_{p_{2}} \cap U_{p_{1}}=\varnothing$. So $\left(\Omega, \tau_{R}(X)\right)$ is $n \beta-T_{2}$.

Theorem 5.18. Let $\left(\Omega, \tau_{R}(X)\right)$ be any nano topological space, $\left(\Lambda, \tau_{R^{\star}}(Y)\right)$ be $n \beta$ -Urysohn nano topological space and $\psi: \Omega \rightarrow \Lambda$ be a quasi nano $\beta$-irresolute. Then its graph $G(\psi)$ is strongly nano $\beta$-closed.

Proof: Let $(x, y) \in \Omega \times \Lambda-G(\psi)$. Then $y \neq \psi(x)$. Since $\Lambda$ is $n \beta$-Urysohn, there exist a $V_{1} \in N \beta O\left(\Lambda, R^{\star}, Y ; \psi(x)\right)$ and a $V_{2} \in N \beta O\left(\Lambda, R^{\star}, Y ; y\right)$ such that $n \beta$ $\operatorname{cl}\left(V_{1}\right) \cap n \beta c l\left(V_{2}\right)=\varnothing$. Again since $\psi$ is quasi nano $\beta$-irresolute, there exists an $U \in N \beta O(\Omega, R, X ; x)$ such that $\psi(U) \subset n \beta c l\left(V_{1}\right) \subset \Omega-n \beta c l\left(V_{2}\right)$. Therefore $\psi(U) \cap n \beta c l\left(V_{2}\right)=\varnothing$. So $G(\psi)$ is strongly nano $\beta$-closed.

Theorem 5.19. Let $\left(\Omega, \tau_{R}(X)\right)$ be $n \beta$-Urysohn possessing the property $n P$ and $\left(\Lambda, \tau_{R^{\star}}(Y)\right)$ be $n \beta$-regular. Let $\psi: \Omega \rightarrow \Lambda$ be nano $\beta$-open bijection. Then $G(\psi)$ is strongly nano $\beta$-closed.

Proof: Let $(x, y) \in \Omega \times \Lambda-G(\psi)$. Then $y \neq \psi(x)$ and so $x \neq \psi^{-1}(y)$. Since $\left(\Omega, \tau_{R}(X)\right)$ is $n \beta$-Urysohn, for each $p \in \psi^{-1}(y)$, there exist a nano $\beta$-open set $V_{x}$ and a nano $\beta$-open set $V_{p}$ containing $x$ and $p$ respectively such that $n \beta$ $\operatorname{cl}\left(V_{x}\right) \cap n \beta c l\left(V_{p}\right)=\varnothing$. Then $\left\{V_{p}: \psi(p)=y\right\}$ is a cover of $\psi^{-1}(y)$ by nano $\beta$-open sets of $\left(\Omega, \tau_{R}(X)\right)$. Since $\psi^{-1}(y)$ is finite, there exist finite number of points 
Separation Axioms and Graphs of Functions in Nano Topological Spaces via Nano

$\beta$-open Sets

$p_{1}, p_{2}, \ldots, p_{k} \in \Omega \quad$ with $\quad \psi\left(p_{i}\right)=y \quad$ for $\quad$ each $\quad i \in\{1,2, \ldots, k\} \quad$ such that $\psi^{-1}(y) \subset \cup_{i=1}^{k} V_{p_{i}}$. Let $G=\cap_{i=1}^{k} V_{x_{i}}$ and $H=\cup_{i=1}^{k} V_{p_{i}}$. Since $\left(\Omega, \tau_{R}(X)\right)$ satisfies the property $n P, n \beta c l(H)=n \beta c l\left(\cup_{i=1}^{k} V_{p_{i}}\right)=\cup_{i=1}^{k} n \beta c l\left(V_{p_{i}}\right)$.

So $n \beta c l(G) \cap n \beta c l(H)=\varnothing$. Again since $\psi$ is nano $\beta$-open and bijective, $\psi(H) \in N \beta O\left(\Lambda, R^{\star}, Y ; y\right)$ and so Theorem 4.5 ensures the existence of an $L \in N \beta O\left(\Lambda, R^{\star}, Y ; y\right) \quad$ such that $n \beta c l(L) \subset \psi(H)$, i.e. $\psi^{-1}(n \beta c l(L)) \subset H$. Therefore $\psi^{-1}(n \beta c l(L)) \cap G=\varnothing$ and thus $\psi(G) \cap n \beta c l(L)=\varnothing$. Thus by Lemma $5.12, G(\psi)$ is strongly nano $\beta$-closed.

\section{Conclusion}

Some researchers $[3,6,8]$ recently have shown that the concept of nano topology can be used as a tool to study some real life problems. Keeping these in mind, we have extended some separation axioms and graphs of functions via nano $\beta$-open sets in nano topology, which may have possible applications in real life situations.

\section{REFERENCES}

1. M.E.Abd El-Monsef, S.N.El-Deeb and R.A.Mahmoud, $\beta$-open sets and $\beta$ -continuous mappings, Bull. Fac. Sci. Assiut Univ., 12(1) (1983) 77-90.

2. D. Andrijevic, Semi-preopen sets, Math. Vesnik, 38 (1986) 24-32.

3. A.A.Azzam, Grill nano topological spaces with grill nano generalized closed sets, Egypt. Math. Soc., 25(2) (2017) 164-166. doi.org/10.1016/j.joems.2016.10.005.

4. M.Lellis Thivagar and C.Richard, On nano forms of weakly open sets, Int. J. Math. Stat. Inven., 1 (1) (2013) 31-37.

5. M.Lellis Thivagar and C.Richard, On nano continuity, Math. Theory Model., 7 (2013) 32-37.

6. M.Lellis Thivagar and Carmel Richard, Nutrition modeling through nano topology, Int. Journal of Engineering Research and Applications, 4(10) (2014) 327-334.

7. D.A.Mary and I.Arockiarani, On b-open sets and b-continuous functions in nano topological spaces, Int. J. Innov. Res. Stud., 3(11) (2014) 98-116.

8. A.A.Nasef, A.I.Aggour and S.M.Darwesh, On some classes of nearly open sets in nano topological spaces, Egypt. Math. Soc., 24 (2016) 585-589.

9. A.Revathy and G.Ilango, On nano $\beta$-open sets, Int. J. Eng. Contemp. Math. Sci., 1(2) (2015) 1-6.

10. M. L.Thivagar and C.Richard, On nano forms of weakly open sets, Int. J. Math. Stat. Invention, 1 (1) (2013), 31-37.

11. M.L.Thivagar and C.Richard, On nano continuity, Math. Theory Model., 7 (2013) 32-37. 